\title{
PROMOVER LA REGULACIÓN DEL COMPORTAMIENTO EN TAREAS DE APRENDIZAJE COOPERATIVO EN LÍNEA A TRAVÉS DE LA EVALUACIÓN
}

\author{
(PROMOTING BEHAVIOR REGULATION IN COOPERATIVE \\ ONLINE LEARNING TASKS THROUGH EVALUATION)
}

Denisse López Benavides

Universitat Oberta de Catalunya (España)

Ibis Álvarez Valdivia

Universitat Autónoma de Barcelona (España)

\section{RESUMEN}

El aprendizaje cooperativo en línea presenta nuevas oportunidades para promover comportamientos regulados que favorezcan la construcción conjunta de conocimientos. Sin embargo, en la práctica docente este aspecto es una preocupación y presenta diversas dificultades. El propósito de este artículo es explicar, desde la perspectiva sociocultural, algunos comportamientos más estratégicos de los estudiantes durante el desarrollo de tareas de aprendizaje cooperativo. Se describe un estudio empírico realizado en un curso en una universidad virtual sobre cuya base se presenta una propuesta metodológica para evaluar y promover comportamientos regulados durante tareas cooperativas con demandas argumentativas en entornos virtuales asincrónicos y escritos.

Palabras clave: aprendizaje cooperativo, evaluación del aprendizaje, aprendizaje regulado, estrategias de enseñanza/aprendizaje, entornos virtuales de aprendizaje, educación superior.

\begin{abstract}
Although online collaborative learning presents new opportunities to promote regulated behaviors to support joint construction of meaning, in teaching practice it can present several difficulties. The purpose of this article is to explain, from a sociocultural perspective, some of the strategies employed by students when engaged in cooperative learning tasks. A virtual university course was studied empirically to evaluate and promote cooperative regulated behavior during argumentative tasks based on written argumentation in virtual and asynchronous communication environments.
\end{abstract}

Keywords: collaborative learning, learning assessment, teaching/learning strategies, regulated learning, online learning environments, higher education. 
Teóricamente, las actividades de aprendizaje diseñadas en formatos colaborativos deben favorecer el intercambio socio-cognitivo entre los estudiantes, incrementar la motivación por el aprendizaje y, consecuentemente, contribuir a elevar la calidad del conocimiento que se construye conjuntamente.

Los estudiantes, mediante interacciones educativas, construyen y reconstruyen información que comparten en el entorno virtual de aprendizaje. La confluencia de ideas permite combinar los aspectos sociales y constructivos durante el proceso de aprendizaje cooperativo, de modo que la integración de las tecnologías debe ser capaz de apoyar ambas cuestiones (Dillenbourg, Schneider y Synteta, 2002; Scardamalia y Bereiter, 1994). Sin embargo, en la práctica esto sigue siendo una preocupación, no siempre se constatan los beneficios de la cooperación al aprendizaje, por otra parte se reconoce la dificultad para evaluar este proceso (Barron, 2003; Gress, Fior, Hadwin y Winne, 2007). Este hecho puede deberse, en parte, a las dificultades para regular el comportamiento que suelen manifestar los estudiantes cuando participan en situaciones de aprendizaje en este tipo de entornos (Borges, 2005; Green, 2001; Harris y Graham, 2006).

Existen diversas teorías que contribuyen a la comprensión de la regulación del aprendizaje cooperativo apoyado con tecnología (CSCL - Computer Supported Collaborative Learning). Desde el enfoque constructivista y socio-cultural del aprendizaje se destacan como ventajas los atributos específicos de la comunicación de base textual que favorecen la reflexión y el discurso crítico (Garrison y Anderson, 2005; Salmon, 2002). No obstante, para que las oportunidades que ofrecen los entornos virtuales de aprendizaje promuevan interacciones entre los alumnos contribuyentes a la construcción conjunta de conocimiento y a la regulación del aprendizaje es preciso cuidar el diseño de las actividades cooperativas. Asimismo en estas actividades parece más importante orquestar y evaluar el proceso que los resultados (Barberà, 2004; Macdonald, 2003; Laurillard, 2009; Onrubia, Colomina y Engel, 2007). En este trabajo se pretende realizar una aportación en este terreno.

En esta línea, el propósito de este artículo es describir algunos indicadores de la regulación del aprendizaje que pueden observarse a lo largo del proceso de cooperación, con la intención de contribuir a la clarificación de los criterios para evaluarlos y promoverlos. Primeramente haremos referencia a las características de la regulación del aprendizaje en entornos de aprendizaje asincrónicos y escritos, a continuación comentaremos brevemente el estudio empírico que da pie a esta propuesta educativa. Por último, y con mayor detenimiento, explicaremos algunos indicadores de diversos modos de regulación de la participación de los estudiantes en 
tareas cooperativas, poniendo el énfasis en la contribución de la función reguladora a la construcción conjunta de significados en este tipo de tareas en entornos virtuales de aprendizaje (EVA).

\section{REGULACIÓN DEL APRENDIZAJE EN TAREAS COOPERATIVAS EN EVA DESDE LA PERSPECTIVA SOCIOCULTURAL DE LA ENSEÑANZA}

El aprendizaje cooperativo es una actividad coordinada y sincrónica, resultado de una tentativa continua de construir y mantener una concepción compartida de un problema. De ahí la naturaleza co-reguladora de la participación en un grupo de trabajo (Lipponen et al., 2002).

Teniendo en cuenta la naturaleza esencialmente social y dialógica de esta modalidad de aprendizaje parece pertinente una aproximación a la concepción socio-constructivista del aprendizaje para su mejor comprensión.

La teoría socio-cultural del aprendizaje procedente de las tesis de Vygotsky (1979) focaliza la importancia del diálogo y de la mediación social (Hung y Der-Thanq, 2001; Wertsch, 1988). Desde esta concepción, en primer término, es preciso distinguir la cooperación de la colaboración. La cooperación concreta la reciprocidad esencial de la colaboración, en función de un diálogo interactivo entre los estudiantes, a través del que comparan y reconstruyen los resultados de sus tentativas individuales para responder al objetivo de una tarea compartida (Roschelle y Teasley 1995).

En este sentido, para la mejor comprensión del proceso de cooperación es imprescindible prestar atención a los formatos interactivos y a cómo estos contribuyen a la regulación del aprendizaje. Muchas concepciones actuales sobre el aprendizaje, especialmente las perspectivas socio-cognitivas y socio-constructivistas que retoman las aportaciones de Vygotsky a la educación, destacan la importancia de la participación del estudiante en la interacción social (Ej. Mercer, 2004; Wegerif, 2006). Esta tesis supone que las personas aprenden conceptos y mejoran sus estrategias de aprendizaje cuando interactúan con otros. Por ejemplo, cuando los estudiantes expresan y defienden sus creencias y opiniones y cuando cuestionan las ideas de los otros, están creando una situación de ayuda para reconocer, clarificar incluso para rectificar inconsistencias en su propio pensamiento.

En este planteamiento es central la idea de que los individuos actúan en contextos sociales donde diversos modos de regulación de la interacción con los pares y docentes determinan la calidad de su participación y los logros de aprendizaje, y es en este plano donde juega un papel fundamental la función reguladora del comportamiento. 
La corregulación, como forma que adquiere la regulación en el aprendizaje cooperativo, pone énfasis en la apropiación gradual de problemas y tareas comunes, compartidos a través de la interacción interpersonal. Es importante atender al hecho de que los mecanismos que se han identificado en las tareas cooperativas hacen referencia a las relaciones psicosociales, interdependencia positiva y construcción conjunta de conocimientos (Johnson y Johnson, 1994; Casanova, Alvarez y Alemany, 2009). Este presupuesto, en relación al estudio de los modos de regulación del aprendizaje cooperativo, advierte de la necesidad de tener en cuenta las diversas modalidades y funciones que puede presentar la regulación del comportamiento de los estudiantes. Cabe entonces preguntarnos cuáles son los modos de regulación que activan o facilitan la manifestación de estos mecanismos y en qué medida contribuye a la construcción conjunta de conocimientos que es, al fin y al cabo, el propósito de la cooperación. A continuación haremos un breve análisis de estas cuestiones.

\section{DIMENSIONES DE LA REGULACIÓN DEL COMPORTAMIENTO DURANTE LA REALIZACIÓN DE TAREAS COOPERATIVAS}

Existen diversos tipos de actuaciones reguladas, más o menos específicas para tareas diferentes, en dominios diferentes, en contextos socioculturales diferentes y para diferentes estudiantes. Asimismo, las actuaciones reguladas son inseparables del propósito de la tarea y del compromiso del estudiante en su realización (Boeckaerts, 2002).

Concretamente, en el contexto de actividades de aprendizaje cooperativas, el proceso de regulación puede oscilar entre situaciones en las que una persona se encarga de suministrar temporalmente información al grupo o de adoptar un rol instructivo (regulación externa), situaciones metacognitivas (autorregulación) y actuaciones co-reguladoras más equilibradas en las que se ven involucrados varios miembros del grupo (Salonen, Vauras, y Efklides, 2005; Volet, Summers y Thurman, 2009). Desde una perspectiva más práctica conviene clarificar estas cuestiones.

- Cooperar exige autorregular el comportamiento en el interior del grupo: es importante tener en cuenta que trabajar efectivamente con otros para resolver una tarea cooperativa depende en parte de la habilidad para autorregular el comportamiento, de ser capaz de buscar y de adoptar ayudas, incluso de saber cuándo y cómo pedir ayuda (Zimmerman y Tsikalas, 2005).

La realización de acciones con base en metas intrínsecas, el pensamiento crítico, el control de las autorreferencias, la elaboración, el pensamiento crítico y la 
solicitud de ayudas de otros compañeros resultan ser estrategias de auto-regulación utilizadas con mayor efectividad por los estudiantes durante tareas cooperativas en entornos virtuales de aprendizaje (Ej. Whipp y Chiarelli, 2004). Acciones como planificar y monitorear la actuación también permiten regular la participación individual en el trabajo cooperativo, incrementan la participación en el diálogo y conduce a mejores resultados globales (Ej. Delfino, Dettori y Persico, 2008; Jermann y Dillenbourg, 2008; Mauri, Colomina y Gispert, 2009).

- La regulación externa puede ser una guía importante en la cooperación: El término regulación externa se aplica a situaciones en las que surge un desnivel de comprensión grupal de algún segmento particular de la tarea cooperativa.

El estudiante, que realiza la regulación externa, es el que se siente mejor familiarizado con ese segmento y quién adopta un papel instructivo con el fin de orientar a los demás a comprender el segmento y así equilibrar el nivel de comprensión en su grupo (Järvelä, Näykki, Laru y Luokkanen, 2007; Manlove, Lazonder y De Jong, 2006; McCaslin, 2009).

- Cooperar exige la corregulación: la regulación compartida (shared regulation) se identifica como el modo más eficaz de regulación, y se refiere a la regulación constante y vigilada de la actividad conjunta, la cual no puede reducirse a una simple actividad individual (Vauras et al., 2003). Este modo de regulación actúa a favor de la interdependencia positiva y activa los lenguajes típicos de la cooperación.

Además de estos formatos típicos de la regulación social durante actividades cooperativas, Weinberger y Fischer (2006) proporcionan un esquema para analizar múltiples dimensiones que se manifiestan durante la construcción de conocimiento en actividades cooperativas con apoyos tecnológicos (CSCL). Entre las dimensiones destacadas por estos autores se encuentran la de la participación, la epistémica, la argumentativa y la de los modos sociales de construcción conjunta.

Esta última dimensión coloca el foco en la dimensión cognitiva de la cooperación, describe en qué medida los estudiantes se refieren a las contribuciones de sus compañeros durante una tarea cooperativa de aprendizaje, un aspecto que ha sido vinculado a la adquisición de conocimientos. Con esta propuesta, los segmentos del discurso pueden ser evaluados con respecto a cómo los alumnos trabajan en la tarea y cómo formulan argumentos desde la cooperación. 
En nuestra opinión, este punto de vista complementa la evaluación del aprendizaje cooperativo, coincidiendo en ello con la propuesta de Anderson et al., (2001), que concibe el razonamiento como fruto de la discusión. Explícitamente estos autores hacen referencia al aprendizaje como un proceso de interiorización, entendido como apropiación participativa, emergente de la interacción social (basado en Rogoff, 1990, Vygotsky, 1979 y Wertsch, 1991). Es por ello que nos parece importante plantear una visión de conjunto de estas cuestiones conceptuales a la hora de categorizar los modos de regulación que pueden observarse durante la realización de una tarea cooperativa (ver figura 1). Así, a la vez que se estudia el proceso de cooperación, para lo cual resultan útiles las dimensiones del discurso cooperativo, se está estudiando el proceso de corregulación que pueda tener lugar a través de la observación de la construcción de conocimientos en un grupo colaborativo en las diferentes fases por las que transcurre (Guanawardena, 1997).

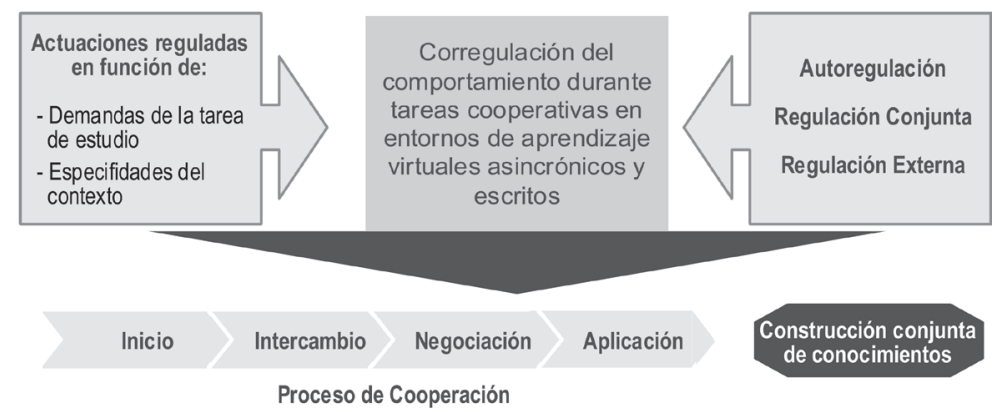

Figura 1. Representación de los modos de regulación durante una actividad cooperativa

En síntesis, en el aprendizaje cooperativo los miembros del grupo representan a agentes autorreguladores interdependientes que constituyen al mismo tiempo una entidad social que crea refuerzos y/o limitaciones para el grupo desde su compromiso individual.

Las diferentes modalidades de regulación se entrelazan de diversas formas durante el proceso cooperativo y se expresan a través de los discursos propios de la cooperación en los entornos virtuales. Así, los lenguajes relacionados con la construcción de conocimiento se concentran en las fases cooperativas de intercambio y de negociación (Casanova, 2008); y los lenguajes relacionados con estrategias reguladoras sociales en las fases de inicio y de aplicación, que corresponden respectivamente con la organización y la realización/presentación del producto final (López, 2009). 
La regulación es entendida así como co-regulación, con atención a un marco conceptual socio-cultural del aprendizaje. Esta perspectiva acentúa el valor de la intersubjetividad y del lenguaje para la gradual apropiación de los objetivos de la tarea que se comparte. En este contexto, la actuación regulada de los estudiantes debe conducir a compartir la responsabilidad de la tarea de aprendizaje -ángulo social de la cooperación- y a la construcción conjunta del conocimiento -ángulo cognitivo-. (Järvenoja y Järvelä, en prensa). Esta doble perspectiva amplía las posibilidades para la intervención educativa durante la realización de actividades cooperativas en tanto ofrece pistas para observar estos mecanismos y para promoverlos a través de una evaluación sistemática y formativa.

En definitiva, teniendo en cuenta estos presupuestos conceptuales, globalmente, queda enfatizada la idea de que la construcción de conocimiento en tareas cooperativas en entornos virtuales asincrónicos y escritos supone la regulación social del proceso de aprendizaje. Más exactamente y ligado con el objetivo de este trabajo, se entiende que la calidad de la corregulación refleja la eficacia de la cooperación para la construcción conjunta de conocimiento, entendido este proceso como un flujo de razonamientos argumentados con base en el diálogo social.

\section{ESTUDIO EMPÍRICO}

La propuesta metodológica que se presenta en este artículo, en aras de contribuir a clarificar e instrumentar la evaluación de la cooperación, tiene su base en un estudio llevado a cabo para explorar a fondo las características de la regulación del comportamiento de estudiantes universitarios mientras realizan tareas de aprendizaje cooperativo con demandas argumentativas, a través de debates críticos en un entorno virtual de comunicación asincrónica y escrita (López, 2009).

Las observaciones realizadas en esta investigación permitieron identificar algunos indicadores de diversos modos de regulación del intercambio social y de la gestión del conocimiento en tareas cooperativas. Correspondiendo a los objetivos que se establecieron se aplicó una metodología de estudio de caso y se analizó el discurso de los estudiantes durante el proceso de trabajo cooperativo. La investigación se llevó a cabo en cinco fases, tal como se muestra en la figura 2. 


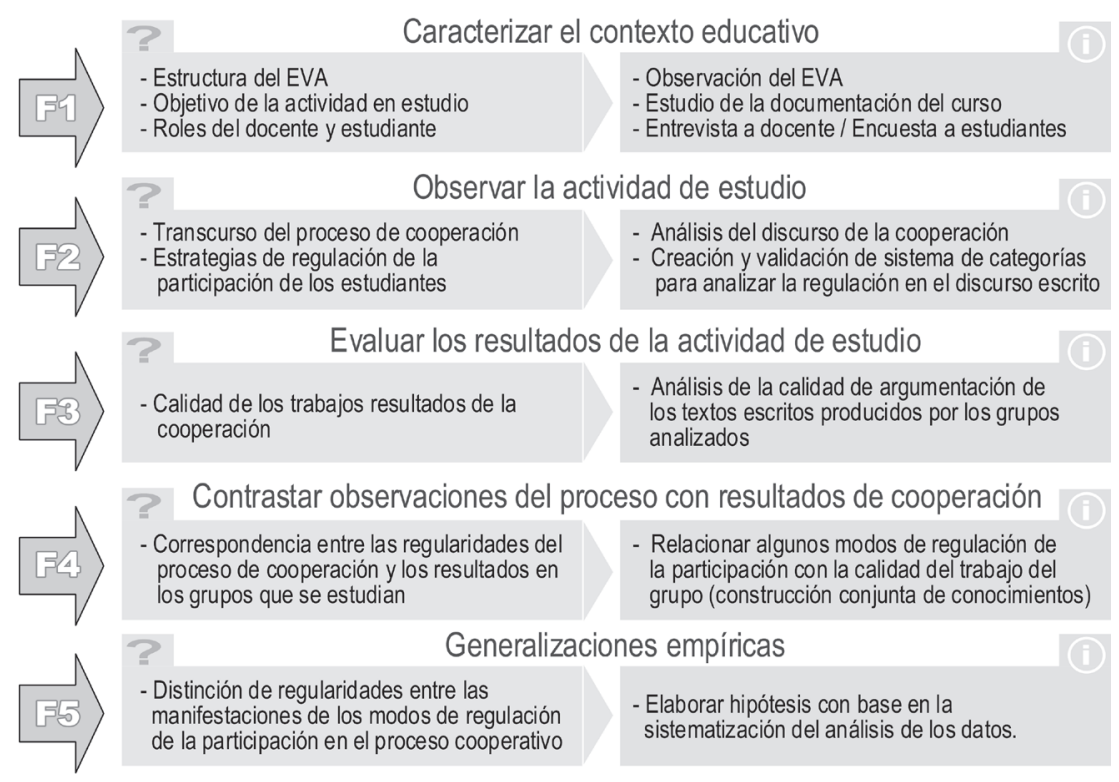

Figura 2. Resumen del diseño del estudio empírico. Fases de la investigación

En la primera fase se realizó una contextualización del estudio. Se conocieron datos importantes relacionados con el entorno virtual en el que se desarrolla la experiencia en estudio, así como los detalles del curso. Se realizaron entrevistas a la docente (una al inicio y otra al final) y una encuesta a los estudiantes, previa al curso, con preguntas abiertas. Los resultados más interesantes en esta fase del estudio fueron las referencias de la docente sobre la actividad de estudio, específicamente la concepción de la actividad conjunta (aprendizaje cooperativo en entornos virtuales) y de la evaluación. De los estudiantes se conocieron sus experiencias y opiniones sobre estrategias eficaces para regular su participación en tareas cooperativas en un EVA, los obstáculos que enfrentaban para la elaboración de textos como tarea de aprendizaje en línea y las estrategias del docente que se consideraban útiles para la realización efectiva de la actividad.

La experiencia se desarrolla en un EVA asincrónico y escrito. La asignatura tiene un diseño estratégico orientado a la sistematización del conocimiento y a impulsar las estrategias para el aprendizaje cooperativo en entornos virtuales (Alvarez y Guasch, 2006) y aplica la técnica evaluativa de co-evaluación. El objetivo general de la asignatura es desarrollar las competencias profesionales que permitan a los estudiantes planificar procesos educativos y formativos con el uso de las TIC, para 
el que se propone el estudio de tres casos reales. La demanda cognitiva de las tareas fue básicamente argumentativa con soporte en la discusión colectiva, llevada a cabo mediante debates críticos virtuales y concretada a través de informes escritos.

Las actividades requirieron de la formación de grupos, realizada a partir de las preferencias individuales por uno de los casos de estudio propuestos. Cada estudiante justificó sus preferencias de acuerdo a su relación con el caso en función de sus experiencias, conocimientos, intereses personales y/o profesionales. Cada grupo dispuso de un espacio de trabajo dentro del área de debate en el aula virtual para llevar a cabo las actividades y recibieron las mismas instrucciones de la docente: documentación con el plan docente y la bibliografía, orientaciones para la lectura comprensiva y la escritura de informes, orientaciones para el desarrollo del debate, pautas para la auto-evaluación y la co-evaluación del proceso, anexos y páginas web para acceder desde el aula virtual.

La docente impartió las instrucciones sobre cada actividad a realizar a través de mensajes al tablón. En los mensajes también se sugirieron pautas para aprovechar el trabajo cooperativo al máximo, haciendo énfasis en la calidad de las intervenciones orientadas hacia la construcción conjunta de conocimiento. El rol de la docente fue principalmente de guía y orientación, prestándose atenta a cualquier inquietud mediante su correo electrónico. Su función, además, fue la de intervenir en situaciones donde requirieron la regulación del intercambio, ya sea con sugerencias y/o aclaraciones sobre la tarea o con refuerzos sociales. También ofrece una retroalimentación grupal o individual, con respecto a la calidad de las contribuciones de cada integrante hacia el grupo en función de la pauta previamente entregada.

Los estudiantes que participaron en este curso disponen de titulaciones profesionales y algunos de ellos, además, cuentan con algún tipo de formación de pos-título o de post-grado.

A través de la encuesta los estudiantes revelaron que, para este tipo de actividad de estudio en EVA habitualmente suelen emplear estrategias de regulación que les permitan consensuar la planificación del trabajo a realizar, así como tratar de conectarse con frecuencia para ver las opiniones de los compañeros y de dejar constancia de lo que cada cual esté realizando en el momento, así como revisar exhaustivamente el requerimiento de la actividad a desarrollar. Además consideraron que se deben asumir diversos roles, proponiendo cronogramas de trabajo y enviando mensajes de motivación sin el ánimo de presionar a nadie. Otras 
estrategias se refirieron a hacer diferentes propuestas para estimular la reacción de otros compañeros, asumir de manera consciente las propuestas sugeridas para incluir en el texto escrito, apoyar a los compañeros de trabajo, dejar constancia sobre las cosas con las que se está de acuerdo y con las que no y proponer alternativas cuando se cree que favorecerán el desarrollo de la tarea.

En cuanto a las estrategias docentes que los estudiantes consideran ayudas para realizar tareas de escritura en línea, hay varios aspectos destacables. Se consideró como necesario recibir instrucciones con pautas y criterios claros respecto a la actividad de estudio y sobre el uso de herramientas colaborativas, todo ello de forma breve, transparente y muy concreto. En cuanto al seguimiento, los estudiantes dieron valor a cuestiones como apoyo, refuerzos sociales y sugerencias si se estimara necesario, comunicación amable pero a la vez exigente y que la retroalimentación del docente se centre en las acciones y en el proceso. Esta información pone de manifiesto la necesidad de regular estratégicamente su participación durante la realización de tareas de aprendizaje en este tipo de entornos.

En la segunda fase de investigación, a través de la observación de la actividad de estudio, se describieron las interacciones durante las tareas cooperativas de escritura. Las observaciones realizadas en buena medida ratificaron las referencias de los estudiantes. Para dar rigor científico a este análisis se creó y se validó un sistema de categorías, con prueba inter-jueces, con las que se exploraron e identificaron los lenguajes correspondientes a las dimensiones de la regulación social y de la regulación cognitiva que ponen en juego los estudiantes a lo largo de la tarea cooperativa. Estas categorías comprenden 14 indicadores que oscilan entre los modos de regulación: auto, externa y conjunta y se pueden ver representadas en cada una de las tres dimensiones del comportamiento.

En la tercera fase investigativa, se evaluó el resultado del aprendizaje de los grupos mediante los informes escritos que elaboraron durante las actividades cooperativas. Para este objetivo se adaptó la propuesta de criterios para evaluar el esquema argumentativo de textos escritos en tareas cooperativas de Reznitskaya et al., (2008) (ver cuadro 1), que permitió contrastar las observaciones del proceso de la cooperación con los resultados, hallándose correspondencias directas. 


\begin{tabular}{|c|c|}
\hline Criterios & Descripción \\
\hline $\begin{array}{l}\text { Relevancia de las ideas } \\
\text { Ajuste de las ideas respecto } \\
\text { al objetivo de la tarea }\end{array}$ & $\begin{array}{l}\text { Ideas introductorias: describe el propósito y/o alcance de las ideas } \\
\text { que se presentan, y se da una breve explicación o resumen de } \\
\text { éste. También puede explicar algunos antecedentes que son } \\
\text { importantes para el posterior desarrollo de las ideas centrales. } \\
\text { Ideas esenciales: posicionamientos centrales directamente } \\
\text { relacionados con la demanda argumentativa de la tarea (criterio } \\
\text { apropiado para valorar el caso). } \\
\text { Ideas no esenciales: posicionamientos centrales no relacionado con } \\
\text { la demanda argumentativa de la tarea (criterio no apropiado para } \\
\text { valorar el caso). }\end{array}$ \\
\hline $\begin{array}{l}\text { Focalización de las ideas } \\
\text { esenciales }\end{array}$ & $\begin{array}{l}\text { Giros Argumentos Relevantes: enunciados razonados e ilustrativos. } \\
\text { Giros Argumentos Circunstanciales: enunciados superficiales poco } \\
\text { o nada ilustrativos. }\end{array}$ \\
\hline $\begin{array}{l}\text { Soporte de las ideas } \\
\text { Fuente de las ideas } \\
\text { que sostienen los } \\
\text { razonamientos }\end{array}$ & $\begin{array}{l}\text { Textual: ideas extraídas más o menos literalmente de las lecturas } \\
\text { previas. } \\
\text { Hipótesis: Enunciados referidos a acciones probables. } \\
\text { Abstracción: Generalización, prescripción sobre cómo actuar o } \\
\text { sobre las consecuencias de la actuación. } \\
\text { Contextualización: Enunciado que reconstruye la situación, con } \\
\text { atención al contexto, la audiencia, etc. }\end{array}$ \\
\hline $\begin{array}{l}\text { Elaboración de las ideas } \\
\text { Esquema argumentativo de } \\
\text { las ideas esenciales }\end{array}$ & $\begin{array}{l}\text { Idea elaborada: Esquema argumentativo bien articulado: Criterio } \\
\text { valorativo + justificación + ejemplos/evidencias + conclusión/ } \\
\text { contextualización de la idea esencial. } \\
\text { Idea incompleta: Giros argumentativos anclados a idea relevante } \\
\text { (criterio valorativo + justificación) pero carente de evidencias o } \\
\text { idea conclusiva contextualizada. } \\
\text { Idea muy incompleta: Criterio poco claro o no justificado, } \\
\text { repeticiones de ideas, giros argumentativos desarticulados o } \\
\text { circunstanciales. }\end{array}$ \\
\hline
\end{tabular}

Cuadro 1. Criterios de evaluación de textos escritos ${ }^{1}$

En una cuarta fase de investigación, se contrastaron las observaciones realizadas en la segunda fase sobre las interacciones durante las tareas cooperativas de escritura con los resultados de la evaluación de los informes escritos de la tercera fase. Este análisis puso en evidencia que los grupos de trabajo que mostraron mejores estrategias para regular sus comportamientos desde la perspectiva social como desde la perspectiva cognitiva, también consiguieron reflejar en sus informes ideas esenciales mejor elaboradas, soportadas con argumentos relevantes en relación con la demanda de la tarea de aprendizaje (valoración crítica de un caso). En los textos mejor estructurados predominaron razonamientos hipotéticos, abstractos y 
contextualizados, que dan cuenta de construcción de significados y de aplicación del conocimiento.

En una última fase se exploraron modelos de interacción, que permitieran observar algunas tendencias en las manifestaciones de las diversas estrategias que utilizan los estudiantes para regular sus intervenciones durante la realización de tareas cooperativas en entornos de aprendizaje virtuales asincrónicos y escritos.

Como resultado de la exploración se identificó un modelo que muestra el comportamiento de las estrategias de regulación a lo largo de la realización de una tarea cooperativa, sugiriendo que las fases son progresivas y que los lenguajes varían en función de la fase de cooperación en la que el grupo se encuentre (ver figura 3).

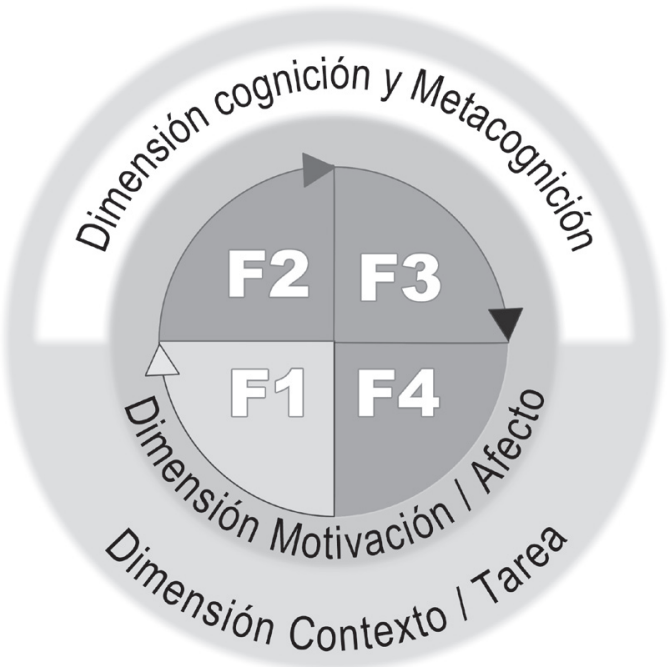

Figura 3. Modelo teórico del proceso de cooperación. Fases de la cooperación

La cooperación de un grupo comienza en el tercer cuadrante, correspondiente a la fase 1 de Inicio. En esta fase la regulación es principalmente de carácter individual, los integrantes del grupo se conocen, se integran y se organizan. Se define la tarea y surgen propuestas para enfocar su realización. Las intervenciones muestran la asunción de compromisos y/o estimulan la integración con los demás miembros del grupo mediante lenguajes que generan diálogo social. En esta fase se identificaron estrategias reguladoras de la dimensión del Contexto/Tarea: Estructurar la tarea, Planificación individual; seguida de la dimensión de Motivación/Afecto: Refuerzos 
sociales; y en una baja frecuencia se observaron lenguajes ligados a la dimensión de la Cognición y Metacognición: Elicitar y Exteriorización.

La segunda fase cooperativa de Intercambio se posiciona en el segundo cuadrante. La regulación en esta fase osciló entre los modos auto y externo. Se observó que el tipo de información intercambiado era acumulativo, y que aunque favorecía positivamente para la realización de la tarea, no eran aportes críticos ni argumentados. En esta fase se evidenciaron reflexiones que relacionaban saberes o experiencias previas con la tarea a realizar, y que valoraban el beneficio que la realización de la tarea podía traer para el contexto cotidiano del estudiante. Las intervenciones muestran la presencia de lenguajes contenidos en indicadores de la dimensión de la Cognición y Metacognición: Elicitar y Exteriorización; y de la dimensión del Contexto/Tarea: Monitorización de su propia participación.

La tercera fase cooperativa de Negociación, ubicada en el primer cuadrante, se caracteriza por la dinámica y regulación grupal. El foco central de la fase es responder al objetivo de la tarea, filtrando la información recolectada de la fase previa, mediante su análisis crítico, con argumentos, propuestas y reflexiones. Las intervenciones se muestran abiertas y flexibles, estimulando el diálogo, la construcción de significados y el consenso grupal. Los indicadores de la regulación observados en esta fase corresponden mayormente a los de la dimensión de la Cognición y Metacognición: Exteriorización, Integración orientada al consenso, Negociar significado y Elicitar; seguidos de la dimensión del contexto/tarea: Monitorización de su propia participación; y de Motivación/Afecto: perspectiva mutua.

En la última fase cooperativa de Aplicación, ubicada en el cuarto cuadrante, se observa también una dinámica y regulación grupal, esta vez el foco central es la aplicación y concreción de lo alcanzado en las fases previas. Se realiza el producto final: informe escrito. Las intervenciones en esta fase expresan constancia y responsabilidad de los miembros para con el grupo, también pueden aparecer aseveraciones cognitivas ilustrando la modificación en los esquemas cognitivos como resultado del proceso de interacción. Los indicadores de regulación en esta fase son de la dimensión de Motivación/Afecto: Refuerzos sociales; de Contexto/ Tarea: Monitorización de su propia participación, Planificación individual; y en una baja frecuencia de la dimensión Cognición y Metacognición: Exteriorización.

En la figura 4 se ilustra, con un ejemplo sencillo (diálogo entre dos participantes de un grupo) como emergen sucesivamente los modos de regulación y los lenguajes que los caracterizan a lo largo de la tarea de aprendizaje cooperativo. 


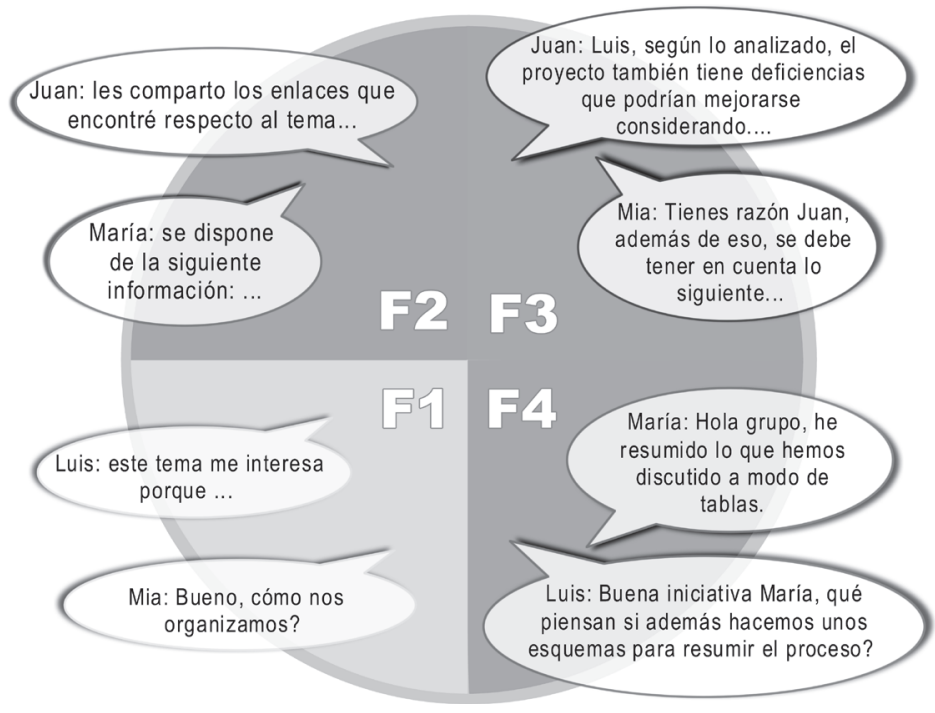

Figura 4. Ejemplos de lenguajes reguladores en las fases de trabajo cooperativo. Fases de la cooperación

La fase cooperativa de mayor interés para la evaluación de la regulación de la construcción del aprendizaje, es la tercera fase como lo propone Casanova (2008). En esta fase, además de evidenciarse la presencia de la corregulación, emergen lenguajes más ligados a la construcción de significado. Esto no representa que las demás fases no sean importantes, al contrario, todas éstas constituyen la base del trabajo cooperativo y las cuatro fases comprenden un solo proceso.

\section{PROPUESTA METODOLÓGICA PARA EVALUAR LA CONSTRUCCIÓN DE CONJUNTA DE CONOCIMIENTOS EN TAREAS COOPERATIVAS EN LÍNEA}

Para evaluar el proceso de la cooperación, haciendo uso de los indicadores de la regulación, es necesario primeramente distinguir las cuatro fases de trabajo cooperativo en los grupos: inicio, intercambio, negociación y aplicación. $\mathrm{Si}$, en cambio, se quiere seguir el trabajo cooperativo de un grupo, es necesario distinguir cada fase cooperativa sobre la marcha. La identificación de las fases permite, por un lado, observar detalladamente el desarrollo de las interacciones de los grupos en el EVA y realizar una evaluación del proceso; y, por otro lado, le permite al docente prever situaciones en las que el trabajo cooperativo se vea amenazado, para que pueda intervenir oportunamente. 
En la actividad cooperativa de debate crítico, es usual que los grupos quieran organizarse por los temas definidos en los objetivos de la tarea, abriendo y siguiendo hilos de discusión concretos. La organización de las intervenciones por estos hilos de discusión favorece al análisis para conocer a fondo la calidad, función, intención y alcance de cada interacción. Las interacciones se componen de intervenciones o mensajes escritos, idealmente aportados por los diferentes miembros del grupo. Cada mensaje contiene unidades temáticas referidas a unidades de significado, pensamiento o ideas que pueden ser identificadas en alguno de los indicadores de la regulación social (ver cuadro 2 y 3 ) o cognitiva (ver cuadro 4).

Los lenguajes en las interacciones, la presencia o ausencia de los indicadores de la regulación pueden orientar a conocer la fase de cooperación por la que los grupos transitan y valorar, si es el caso, si hay aprendizaje cooperativo o no en el trabajo grupal.

Sobre esta base conceptual a continuación se resumen los indicadores para la observación de la regulación extraídos del estudio empírico que se describió en el epígrafe precedente. Para su mejor comprensión los indicadores se organizan según la dimensión del comportamiento definidos para la investigación. Los cuadros definen las estrategias para la regulación del comportamiento que parecen más eficaces en cada fase del aprendizaje cooperativo. Se describen y se ejemplifican las estrategias más efectivas en cada una de las dimensiones por las que transcurre la regulación del aprendizaje.

Las dimensiones de Motivación/Afecto y Contexto/Tarea contienen los mecanismos reguladores esenciales de la cooperación que dan soporte a la construcción conjunta durante las cuatro fases del trabajo cooperativo. Para regular los aspectos motivacionales durante la realización de tareas cooperativas son esenciales actuaciones que permitan la orientación del esfuerzo a metas de logros, el manejo de las expectativas de éxito y fracaso a través de diversos refuerzos, las auto-percepciones de competencia y habilidad para realizar la tarea, las expresiones sobre el valor asignado a la tarea así como muestras afectivo-emocionales que garanticen un confortable clima de trabajo en grupo. En el cuadro 2 se describen estas actuaciones, más típicas en las fases inicial y final de la tarea cooperativa. 


\begin{tabular}{|c|c|}
\hline \multicolumn{2}{|c|}{ Fases Inicial y Final de la tarea cooperativa } \\
\hline \multicolumn{2}{|l|}{ Regulación externa a través de refuerzos sociales } \\
\hline \multicolumn{2}{|c|}{$\begin{array}{l}\text { Intervenciones emotivas que respaldan ideas o actuaciones de otros porque inciden positiva- } \\
\text { mente en la cognición o en la motivación de los demás. }\end{array}$} \\
\hline $\begin{array}{l}\text { Fase inicial: "Es genial repetir con quien ya he trabajado y mi } \\
\text { mas cordial bienvenida a los que nos pondremos a ello". }\end{array}$ & $\begin{array}{l}\text { Fase final: "Gracias por el trabajo } \\
\text { realizado, en particular a Camila y } \\
\text { a Juan. Sin vuestro tirón final no lo } \\
\text { habríamos conseguido". }\end{array}$ \\
\hline \multicolumn{2}{|c|}{$\begin{array}{l}\text { Regulación conjunta a través de perspectiva mutua } \\
\text { Interacciones que comunican un acuerdo recíproco, se reconoce, se valora y se refuerza una idea. }\end{array}$} \\
\hline $\begin{array}{l}\text { Fase inicial: "Hola Grupo, estoy de acuerdo con las fechas } \\
\text { propuestas por Carlos y con la organización para la } \\
\text { planificación deMarta. Asícadacualpuede organizarse mejor". }\end{array}$ & $\begin{array}{l}\text { Fase final: "Completamente de } \\
\text { acuerdo con Diana y Juana. La } \\
\text { forma como nos organizamos y el } \\
\text { compromiso de todos fue lo que } \\
\text { nos permitió sacar adelante el } \\
\text { proyecto". }\end{array}$ \\
\hline
\end{tabular}

Cuadro 2. Dimensión Motivación / Afecto

Los aspectos relativos al contexto de aprendizaje se regulan mejor a través de intervenciones que permitan la percepción de las características de la tarea y del contexto en el que tiene lugar la actividad, que clarifiquen la estructura del trabajo y los métodos recomendables para realizar la tarea (ver cuadro 3).

En general, las actuaciones más estratégicas en este sentido son aquellas que llaman la atención sobre las pautas e intervenciones del docente y del tipo de interacciones que se establece entre alumnos y docentes. Estos modos de regulación favorecen el desarrollo de la tarea cooperativa sobre todo en la fase inicial y final. 


\begin{tabular}{|c|c|}
\hline \multicolumn{2}{|c|}{ Fases Inicial y Final de la tarea cooperativa } \\
\hline \multicolumn{2}{|c|}{$\begin{array}{l}\text { Autorregulación planificando la participación } \\
\text { El estudiante valora los recursos y tiempo disponibles para determinar su colaboración y asume } \\
\text { voluntariamente responsabilidades. }\end{array}$} \\
\hline $\begin{array}{l}\text { Fase inicial: "Deciros que este fin de semana no tendré } \\
\text { acceso a internet, por tanto no me puedo encargar de } \\
\text { elaborar el plan de trabajo". }\end{array}$ & $\begin{array}{l}\text { Fase final: "Me ofrezco para darle el } \\
\text { formato final al documento y entregarla } \\
\text { mañana si queréis". }\end{array}$ \\
\hline \multicolumn{2}{|l|}{$\begin{array}{l}\text { Autorregulación a través del monitoreo de su participación } \\
\text { Control de la gestión de su propia participación. }\end{array}$} \\
\hline $\begin{array}{l}\text { Fase inicial: "Estos días he estado bastante desconectada } \\
\text { de esta asignatura, sin embargo me he puesto al tanto de } \\
\text { lo que se ha aportado". }\end{array}$ & $\begin{array}{l}\text { Fase final: "Como les había prometido, les } \\
\text { adjunto hoy las modificaciones que le he } \\
\text { hecho al documento final". }\end{array}$ \\
\hline \multicolumn{2}{|c|}{$\begin{array}{l}\text { Regulación externa para estructurar la tarea } \\
\text { Intervenciones con preguntas o propuestas sobre organización, procedimiento, roles, recursos, } \\
\text { planificación temporal, formato del texto, etc. }\end{array}$} \\
\hline $\begin{array}{l}\text { Fase inicial: "Creo que tenemos que fijar una fecha para } \\
\text { decidir en qué vamos a centrarnos, y sugiero que sea el } \\
\text { lunes 5". }\end{array}$ & $\begin{array}{l}\text { Fase final: "Luis, tenemos dos días para } \\
\text { entregar, así que ya se le puede dar el } \\
\text { formato final al documento..." }\end{array}$ \\
\hline \multicolumn{2}{|c|}{$\begin{array}{l}\text { Regulación externa explicitando la tarea } \\
\text { Interacciones no argumentativas que giran alrededor del objetivo común. El objetivo de la tarea } \\
\text { se analiza, se aclara, se reformula, se repasa. }\end{array}$} \\
\hline $\begin{array}{l}\text { Fase Inicial: "Mónica, releyendo la tarea, pienso que lo } \\
\text { más conveniente sería que cada uno vaya aportando } \\
\text { ideas e informaciones sobre los interrogantes que se nos } \\
\text { plantea y luego generamos una síntesis de cada cuestión". }\end{array}$ & $\begin{array}{l}\text { Fase final: "Si, Juan, al fin hemos tocado } \\
\text { todos los temas que se nos pedía acotar". }\end{array}$ \\
\hline \multicolumn{2}{|c|}{$\begin{array}{l}\text { Regulación conjunta interpelando la responsabilidad/participación de los demás } \\
\text { Interacciones que solicitan la ayuda/colaboración a sus compañeros, en función de la } \\
\text { organización y realización de la tarea. }\end{array}$} \\
\hline $\begin{array}{l}\text { Fase inicial: "Bueno, ahora que ya estamos todos, ¿cómo } \\
\text { nos organizamos?" }\end{array}$ & $\begin{array}{l}\text { Fase final: "Hay unos comentarios que } \\
\text { creo son para Sergio y están relacionados } \\
\text { con lo que Teresa también menciona } \\
\text { respecto al manejo de citas textuales". }\end{array}$ \\
\hline
\end{tabular}

Cuadro 3. Dimensión: Contexto y Tarea

Por último, en esta propuesta, la construcción del conocimiento en el trabajo cooperativo corresponde con los mecanismos reguladores de la dimensión Cognición y Metacognición (ver cuadro 4). Para esta dimensión se destacan estrategias de regulación cognitivas y metacognitivas útiles para la activación de conocimiento previo, para situar la tarea de aprendizaje y para coordinar el trabajo del grupo en función de mantener la necesaria interdependencia. 


\begin{tabular}{|l|}
\multicolumn{1}{|c|}{ Fases de Intercambio y de Negociación } \\
\hline $\begin{array}{l}\text { Autorregulación con base en la auto-evaluación } \\
\text { Intervenciones que reflejan la valoración de sus conocimientos o experiencias previas que puedan } \\
\text { contribuir a la resolución de la tarea o que reflejen lo que la resolución de la tarea le aportará } \\
\text { respecto a las demandas de su contexto cotidiano. }\end{array}$
\end{tabular}

Fase intercambio: "Yo creo que con las tareas propuestas para el curso mis competencias para la comunicación e interacción van a mejorar".
Fase negociación: "Hola grupo, tuve una experiencia similar a la que se plantea en el estudio de caso en la que actué de la siguiente manera..."

\section{Autorregulación situando el propio proceso de aprendizaje}

El estudiante asimila el objetivo de la tarea, lo relaciona con sus conocimientos previos y valora lo que necesita para alcanzarlo.

Fase intercambio: "Con respecto al objetivo de Fase negociación: "Creo que hay que analizar a la tarea, comentaros que yo vi algo bastante relacionado con esto en otro curso y pienso que se lo podría adaptar sobre este contexto". fondo lo que se ha aportado hasta ahora. Hay algunas ideas que no son coherentes, con lo que se nos ha pedido realizar."

Autorregulación a través del monitoreo de su participación

Control de la gestión de su propia participación.

Fase negociación: "Hola Grupo, en atención Fase intercambio: "Rosa, acabo de terminar una al compromiso adquirido, de avanzar en las primera lectura del documento que nos compartes, reflexiones sobre algunos tópicos que No hemos en breve hago conocer mis opiniones al respecto". abordado hasta ahora, comparto con Ustedes unas reflexiones sobre..."

\section{Regulación conjunta a través de consenso corto y rápido}

Interacciones que muestran conformidad o neutralidad con algo expuesto.

Fase intercambio: "Hola! Pues como queráis, modelo pedagógico o modelo de evaluación..." Fase negociación "Realmente tu argumento me convence. No tengo nada en contra".

\section{Autorregulación a través de la exteriorización}

Intervenciones no argumentativas que aportan información de contenido textual o expresan puntos de vista sobre el contenido a tratar, sin referencias a previas contribuciones.

Fase intercambio: "Desde mi punto de vista creo que el Caso 3 cuenta con más fortalezas que debilidades. A continuación enumero algunas de ellas".

Fase negociación: "Por lo que se refiere a la capacitación y concienciación de los empleados creo que, aunque haya deficiencias, las acciones llevadas a cabo son muy útiles y apropiadas".

\section{Regulación conjunta a través de la elicitación}

Intervenciones que solicitan, directa o indirectamente, la reacción de otro compañero(a) en función del contenido de la tarea.

Fase intercambio: "Para entrar en contexto, quisiera que me ayudaran a aclarar lo siguiente: a qué se refiere formación universitaria de primer y tercer ciclo, existe algún segundo ciclo?"
Fase negociación: "Gracias, Carlos! ¿Creéis que es necesario desarrollar esos cuestionarios o basta con describir cómo deberían ser?" 


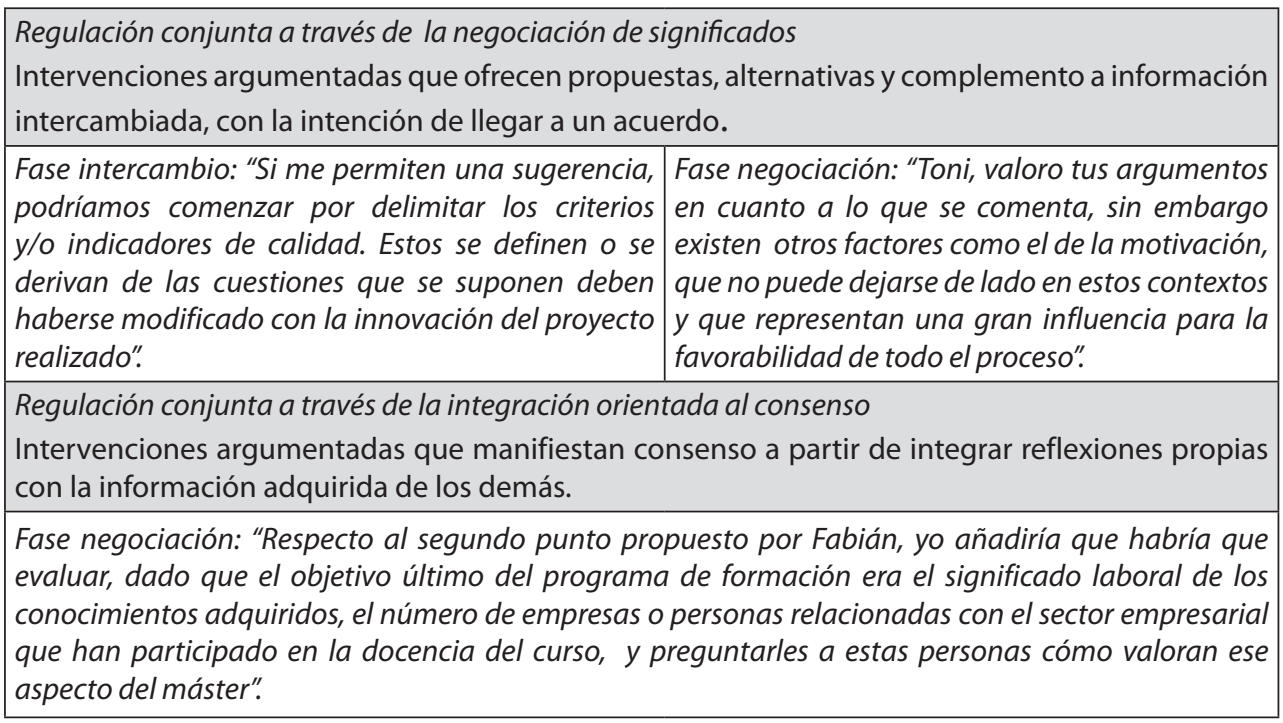

Cuadro 4. Dimensión Cognición y Meta-cognición

Estos aspectos, como subraya Zimmerman (1989), constituyen un sistema que involucra factores más personales "self", factores derivados de sus actuaciones, y a los factores externos, relativos al contexto o entorno de aprendizaje. En la situación de aprendizaje, diversos componentes de la tarea y del contexto se integran y configuran un tipo de acción regulada pertinente para el propósito de la tarea. Por lo tanto, el propósito de la tarea, ya sea de aprendizaje o de evaluación, puede de antemano indicar el tipo de regulación que se desea promover y, con ello, diseñar entornos y situaciones de aprendizaje que proporcionen estímulos y soportes adecuados a la función que ha de tener la regulación del aprendizaje, de acuerdo con el propósito de la actividad de estudio.

\section{COMENTARIOS FINALES}

Mediantela identificación delosindicadores dela regulación en las intervenciones de las interacciones de los grupos durante cada una de las fases cooperativas, es posible, por un lado, evaluar el desarrollo de las interacciones de los grupos en el EVA y realizar una evaluación del proceso; y, por otro lado, prevenir situaciones desfavorables para el trabajo cooperativo, mediante la oportuna intervención docente. 
Las nuevas tecnologías han estimulado una explosión creativa de nuevas maneras para enseñar y para aprender, aunque las TIC en sí mismas raramente son diseñadas con un fin de enseñanza y aprendizaje. Es por ello que, para conseguir mejores resultados de las TIC para la educación, necesitamos estudiar detenidamente las particularidades que adquieren los procesos de enseñanza y aprendizaje cuando se introducen aplicaciones tecnológicas.

Desde la investigación necesitamos construir un marco conceptual pedagógico que permita responder al desafío que supone para docentes y estudiantes, de modo que enseñar y aprender con las tecnologías conlleve realmente a mejorar las experiencias de aprendizaje. Deseamos con este trabajo sentar bases en este sentido, sabiendo de antemano que la puesta en práctica de esta propuesta dependerá ante todo de su discusión constructiva y contextualizada. La formación docente puede ser un buen escenario para este propósito.

\section{NOTAS}

1 Categorías del esquema argumentativo de texto escrito en tarea cooperativa. Adaptado de Reznitskaya et al. (2008).

\section{REFERENCIAS BIBLIOGRÁFICAS}

Álvarez, I.; Guasch， T. (2006). Diseño de estrategias interactivas para la construcción de conocimiento profesional en entornos virtuales de enseñanza y aprendizaje. Revista de Educación a Distancia, RED. Año V, Número 14 [en línea] Disponible en: http://www.um.es/ead/red/14/ (consulta 2006, 31 de marzo).

Anderson, R. C.; Nguyen-Jahiel, K.; McNurlen, B.; Archodidou, A.; Kim, S.; Reznitskaya, A.; et al. (2001). The snowball phenomenon: Spread of ways of talking and ways of thinking across groups of children. Cognition and Instruction, 19 (1), (1-46).

Barberá, E. (2004). La educación en la red. Actividades de enseñanza y aprendizaje. España: Paidós.

Barron, B. (2003). When smart groups fail. Journal of the Learning Sciences, 12, (307-359).
Boeckaerts, M. (2002). Bringing about change in the classroom: Strenghts and weaknesses of the self regulated learning approach. Learning and Instruction, 12, (589-604).

Borges, F. (2005). La frustración del estudiante en línea. Causas y acciones preventivas. Digithum. UOC, 7. [en línea] Disponible en: http://www.uoc. edu/digithum/7/dt/esp/borges.pdf (consulta 2009, 7 de julio).

Casanova, M. (2008). Aprendizaje cooperativo en un contexto virtual universitario de comunicación asincrónica: un estudio sobre el proceso de interacción entre iguales a través del análisis del discurso. Tesis Doctoral. Universidad Autónoma de Barcelona. [en línea] Disponible en: http://www.tdx. cat/TDX-0331109-134502/ (consulta 2009, 6 de julio). 
Casanova, M.; Álvarez, I.; Alemany, I. (2009). Propuesta de indicadores para evaluar y promover el aprendizaje cooperativo en un debate virtual. EDUTEC, Revista Electrónica de Tecnología Educativa, 28. [en línea] Disponible en: http:// edutec.rediris.es/Revelec2/revelec28/ edutec 28 indicadores evaluar aprendizaje.html (consulta 2009, 6 de julio).

Delfino, M.; Dettori, G.; Persico, D. (2008). Self-regulated learning in virtual communities Technology, Pedagogy and Education, 17 (3), (195-205).

Dillenbourg, P.; Schneider, D. K.; Synteta, P. (2002). Virtual learning environments. In: Dimitracopoulou, A. (Ed.), Proceedings of the 3rd Hellenic Conference on Information and Communication Technologies in Education (3-18). Greece: Kastaniotis Editions.

Garrison, D.; Anderson, T. (2005). El e-learning en el siglo XXI: investigación $y$ práctica (trad. de A. Fuentes). Barcelona: Octaedro. [V.O.: E-learning in the 21st century. RoutledgeFalmer, 2003].

Green, S. (2001). A study of the effects of content and structural support in writing tasks. 12th European Conference on Reading. Dublin, Ireland.

Gress, C. L.; Fior, M.; Hadwin, A. F.; Winne, P. H. (2007) Measurement and assessment in computer-supported collaborative learning. Computers in Human Behavior, DOI:10.1016/j. chb.2007.05.012.

Harris, K.; Graham, S. (2006). Improving the writing, knowledge, and motivation of struggling young writers: Effects of self-regulated strategy development with and without peer support. American Educational Research Journal, 43 (2), (295-340).

Hung, D.; Der-Thanq, Ch. (2001). Situated cognition, Vygotskian thought and learning from the communities of practice perspective: implications for the design of web-based E-learning. Educational Media International, 38 (1), (3-12).

Järvelä, S.; Näykki, P., Laru, J.; Luokkanen.; T. (2007). Structuring and Regulating Collaborative Learning in Higher Education with Wireless Networks and Mobile Tools. Educational Technology y Society, 10 (4), (71-79).

Järvenoja, H.; Järvelä, S. (in press). Emotion control in collaborative learning situations-Dostudents regulateemotions evoked from social challenges? British Journal of Educational Psychology. DOI: 10.1348/000709909X402811.

Jermann, P.; Dillenbourg, P. (2008). Group mirrors to support interaction regulation in collaborative problem solving. Computers y Education, 51, (279-296).

Johnson, R.; Johnson, D. (1994). An overview of cooperative learning. En: Thousand, J.; Vila, R.; Nevin, A. Creativity and collaborative learning. A practical guide to empowering students and teachers. Baltimore: Paul H. Brookes.

LaPointe, D.; Gunawardena, N. (2004). Developing, Testing and Refining of a Model to Understand the Relationship between Peer Interaction and Learning Outcomes in Computer-Mediated Conferencing. Distance Education, 25 (1), (83-106).

Laurillard, D. (2009). The pedagogical challenges to collaborative technologies. Computer-Supported Collaborative Learning, 4, (5-20).

Lipponen, L.; Rahikainen, M.; Hakkarainen, K.; Palonen, T. (2002). Effective participation and discourse through a computer network: Investigating elementary students' computersupported interaction. Journal of Educational Computing Research, 27, (353-382).

López, D. (2009). Regulación del comportamiento durante la construcción conjunta de conocimientos 
en tareas cooperativas en entornos de aprendizaje virtuales asincrónicos $y$ escritos. Trabajo Final de Investigación. Máster en Educación y TIC (e-learning). Universitat Oberta de Catalunya.

Macdonald, J. (2003). Assessing online collaborative learning: process and product. Computers y Education, 40 (4), (377-391).

Manlove, S.; Lazonder, A.; De Jong, T. (2006). Regulative support for collaborative scientific inquiry learning. Journal of Computer Assisted Learning, 22 (2), (87-98).

McCaslin, M. (2009). Co-Regulation of Student Motivation and Emergent Identity Educational Psychologist, 44 (2), (137-146).

Mercer, N. (2004). Sociocultural discourse analisys: analysing classroom talk as a social mode of thinking. Journal of Applied Lingüístics, 1 (2), (137-168).

Onrubia, J.; Colomina, R.; Engel, A. (2007). Los entornos virtuales de aprendizaje basados en el trabajo en grupo y el aprendizaje colaborativo. En: Coll, C.; Monereo, C. (Eds.) (2007). Psicología de la Educación virtual. Aprender y enseñar con las Tecnologías de la Información y la Comunicación (233252). Madrid: Morata.

Reznitskaya, A.; Kuo, L.-J.; Glina, M.; Anderson, R. (2008). Measuring argumentative reasoning: What's behind the numbers? Learning and Individual Differences, 19 (2), (219-224).

Rogoff, B. (1990).Apprenticeship in thinking: Cognitive development in social context. New York: Oxford University Press.

Roschelle, J.; Teasley, S. D. (1995). The construction of shared knowledge in collaborative problem solving. In: O'Malley, C. (Ed.), Computer-supported collaborative learning (69-97). Berlin: Springer.

Salmon, G. (2002). Mirror, Mirror, on my screen... Exploring online reflections
The British Journal of Educational Technology, 33 (4), (383-396).

Salonen, P.; Vauras, M.; Efklides, A. (2005). Social interaction-What can it tell us about metacognition and coregulation of learning? European Psychologist, 10, (199-208).

Scardamalia, M.; Bereiter, C. (1994). Computer support for knowlege-building communities. Journal of the Learning Sciences, 3 (3), (265-283).

Vauras, M.; Iiskala, T.; Kajamies, A.; Kinnunen, R.; Lehtinen, E. (2003). Shared-regulation and motivation of collaborating peers: a case analysis. Psychologia: An International Journal of Psychology in the Orient, 46, (19-37).

Volet, S.; Summers, M.; Thurman, J. (2009). High-level co-regulation in collaborative learning: How does it merge and how is it sustained, Learning and Instruction, 19 (2), (128-143).

Vygotsky, L. (1979) Consciousness as a problem in the psychology of behaviour. Soviet Psychology, 17, (3-35).

Wegerif, R. (2006). A dialogic understanding of the relationship between CSCL and teaching thinking skills. International Journal of Computer-Supported Collaborative Learning, 1, (143-157).

Weinberger, A.; Fischer, F. (2006). A framework to analyze argumentative knowledge construction in computersupported collaborative learning. Computers y Education, 46, (71-95).

Wertsch, J. (1988). Vygotsky y la formación social de la mente. Barcelona: Paidós.

Wertsch, J. (1991). Voices of the mind: A sociocultural approach to mediated action. Cambridge, MA: Harvard University Press.

Whipp, J.; Chiarelli, S. (2004). Self-regulation in a web-based course: A case study. Educational Technology Research and Development, 52 (4), (5-22).

Zimmerman, B.; Tsikalas, K. (2005). Can computer-based learning environments (CBLEs) be used as self-regulatory 
tools to enhance learning? Educational

Psychologist, 40, (267-271).

Zimmerman, B. (1989). A social cognitive view of self-regulated academic learning. Journal of Educational Psychology, 81 (3), (329-339).

\section{PERFIL ACADÉMICO Y PROFESIONAL DE LAS AUTORAS}

Denisse López Benavides. Graduada de la Universidad de Bremen en Informática de los Medios con foco en la concepción, diseño, creación y desarrollo de CBT's (Computer Based Training) y WBT's (Web Based Training). Máster en Creación y Producción Multimedia:Tecnologías y aplicaciones de la UOC y Máster Internacional de Educación y las TIC (E-learning).

E-mail: dlopezben@uoc.edu

Ibis Marlene Álvarez Valdivia. Doctora en Psicología y profesora de la Facultad de Psicología de la Universidad Autónoma de Barcelona (UAB) y consultora de la Universidad Abierta de Cataluña (UOC). Integra el Seminario Interuniversitario de Investigación en Estrategias de Enseñanza y Aprendizaje (SINTE) http://www. sinte.es/joomla cast/

E-mail: ibismarlene.alvarez@uab.cat

DIRECCIÓN DE LAS AUTORAS:

Universitat Autonòma de Barcelona

Facultad de Ciencias de la Educación.

Edifici G. 08193

Bellaterra (Cerdanyola del Vallès). España

Fecha de recepción del artículo: 23/07/09

Fecha de aceptación del artículo: 14/04/10 